\title{
Well-posedness and attainability of indefinite stochastic linear quadratic control in infinite time horizon
}

\author{
M. Ait Rami ${ }^{\mathrm{a}}$, Xun Yu Zhou ${ }^{\mathrm{a}, *}$, J.B. Moore ${ }^{\mathrm{b}}$ \\ a Department of Systems Engineering and Engineering Management, The Chinese University of Hong Kong, Shatin, Hong Kong \\ ${ }^{\mathrm{b}}$ Department of Systems Engineering, Research School of Information Science and Engineering, The Australian National University, \\ Canberra, ACT 0200, Australia
}

Received 27 July 1999; received in revised form 2 May 2000; accepted 22 May 2000

\begin{abstract}
This paper is concerned with a stochastic linear-quadratic (LQ) problem in an infinite time horizon with multiplicative noises both in the state and the control. A distinctive feature of the problem under consideration is that the cost weighting matrices for the state and the control are allowed to be indefinite. A new type of algebraic Riccati equation - called a generalized algebraic Riccati equation (GARE) - is introduced which involves a matrix pseudo-inverse and two additional algebraic equality/inequality constraints. It is then shown that the well-posedness of the indefinite LQ problem is equivalent to a linear matrix inequality (LMI) condition, whereas the attainability of the LQ problem is equivalent to the existence of a "stabilizing solution" to the GARE. Moreover, all possible optimal controls are identified via the solution to the GARE. Finally, it is proved that the solution to the GARE can be obtained via solving a convex optimization problem called semidefinite programming. (C) 2000 Elsevier Science B.V. All rights reserved.
\end{abstract}

Keywords: Stochastic linear-quadratic control; Well-posedness; Attainability; Generalized algebraic Riccati equation; Maximal solution; Stabilizing solution; Linear matrix inequality; Semidefinite programming

\section{Introduction}

During the past decades, linear-quadratic (LQ) control theory based on Riccati equations, both for deterministic $[9,14,16]$ and stochastic $[17,4,7]$ systems, has developed into a major research field in control theory and has found tremendous applications for solving real world problems. In the LQ literature, it is typically assumed that the cost function has a positive-definite weighting matrix for the control term and a nonnegative definite weighting matrix for the state term. In fact, the nonnegative definiteness of the control cost matrix is necessary for the well-posedness of the deterministic LQ problem.

However, it was found in [6] for the first time that a stochastic LQ problem with indefinite control cost may still be well-posed. This phenomenon, which occurs when the diffusion term depends on the control, has to do with the deep nature of the uncertainty involved. To intuitively explain this, let us first recall the reason why a deterministic LQ problem with a negative control cost matrix becomes ill-posed. Indeed, in this

\footnotetext{
* Corresponding author. Tel.: 852-2609-8320; fax: 852-2603-5505.

E-mail address: xyzhou@se.cuhk.edu.hk (X.Y. Zhou).
} 
case one can arbitrarily increase the control level, so as to arbitrarily reduce the total control cost, and hence the cost function value, to negative infinity. Thus the problem is trivial and meaningless. Contrary to this, in a stochastic LQ problem where the control influences the volatility, even if the control cost is negative, increasing the control level to infinity may not result in a negatively infinite cost. The reason is that while increasing the control will reduce the control cost, it will also enlarge the uncertainty in the system (because the diffusion term is affected by the control) which is essentially costly. Therefore, one has to strike a balance between the gain due to a larger control and the loss due to a greater risk, which, in turn, gives rise to a meaningful and well-posed problem.

In [6], the indefinite stochastic LQ problem was studied for the case of continuous-time, single-objective, and finite time horizon. Subsequent researches include extensions to the cases of discrete-time [12], multi-objective [11], and infinite time horizon [1]. In all these works the standard matrix inverse is involved in the Riccati equation, requiring the related term to be nonsingular. Applications of indefinite LQ problems can be found in [6] for pollution control, in [18] for portfolio selection, and in [10] for hedging a contingent claim.

In this paper, we study an indefinite stochastic LQ problem in the infinite time horizon, without assuming nonsingularity of any matrix involved. A new type of algebraic Riccati equation - called a generalized algebraic Riccati equation (GARE) - is introduced which involves a matrix pseudo-inverse and two additional algebraic equality/inequality constraints. Since now the LQ problem is indefinite, the well-posedness of the problem becomes the first important issue. We derive the necessary and sufficient condition for the well-posedness in terms of a so-called linear matrix inequality (LMI) condition that is easier to handle computationally $[5,15]$. Moreover, the attainability of the LQ problem, namely, the existence of an optimal control, is investigated via the GARE. It turns out that the solvability of the GARE is necessary for the attainability but not sufficient. This is due to the nature of an infinite time horizon problem, which requires a meaningful control to be stabilizing. Therefore, we further introduce the notion of a stabilizing solution to the GARE and show that the attainability of the LQ problem is equivalent to the existence of a stabilizing solution to the GARE.

The rest of the paper is organized as follows. In Section 2, we formulate the indefinite LQ problem and give necessary preliminaries. Section 3 is devoted to the well-posedness of the problem whereas Section 4 to the attainability. In Section 5, we discuss solving the LQ problem via a convex optimization problem. Some illustrative examples are given in Section 6. Finally, Section 7 concludes the paper.

\section{Problem formulation and preliminaries}

We make use of the following notation throughout the paper:

$M^{\prime}:$ the transpose of a matrix $M$;

$\operatorname{Tr}(M)$ : the sum of diagonal elements of a square matrix $M$;

$|M|:=\sqrt{\operatorname{Tr}\left(M M^{\prime}\right)}$;

$\mathscr{S}^{n}:$ the space of all $n \times n$ symmetric matrices;

Ex: Expected value of a random variable $\mathrm{x}$.

\subsection{Problem formulation}

Let $\left(\Omega, \mathscr{F}, \mathscr{P} ; \mathscr{F}_{t}\right)$ be a given filtered probability space with a standard one-dimensional Brownian motion $w(t)$ on $[0,+\infty)$ (with $w(0)=0$ ). The Brownian motion is assumed to be one dimensional only for simplicity; there is no essential difference with the multi-dimensional case. Define the following set:

$$
L_{2}^{\text {loc }}\left(\mathbf{R}^{k}\right)=\left\{\begin{array}{l}
\phi(\cdot):[0,+\infty) \times \Omega \rightarrow \mathbf{R}^{k} \mid \phi(\cdot) \text { is } \mathscr{F}_{t} \text {-adapted, measurable, } \\
\text { and } \quad \mathbf{E} \int_{0}^{T}|\phi(t, \omega)|^{2} \mathrm{~d} t<+\infty, \forall T \geqslant 0
\end{array}\right\}
$$


The system under consideration is described by the following Itô stochastic differential equation:

$$
\begin{aligned}
& \mathrm{d} x(t)=[A x(t)+B u(t)] \mathrm{d} t+[C x(t)+D u(t)] \mathrm{d} w(t), \\
& x(0)=x_{0} \in \mathbf{R}^{n},
\end{aligned}
$$

where $A, B, C$ and $D$ are real matrices of dimension $n \times n, n \times n_{u}, n \times n$ and $n \times n_{u}$, respectively. A process $u(\cdot)$ is called a control if $u(\cdot) \in L_{2}^{\text {loc }}\left(\mathbf{R}^{n_{u}}\right)$. A control $u(\cdot)$ is called (mean-square) stabilizing (w.r.t. $\left.x_{0}\right)$ if the corresponding state $x(\cdot)$ of (1) with the initial state $x_{0}$ satisfies $\lim _{t \rightarrow+\infty} \mathbf{E}\left[x(t)^{\prime} x(t)\right]=0$. A feedback control $u(t)=K x(t)$, where $K$ is a constant matrix, is called stabilizing if for every initial state $x_{0}$, the corresponding state $x(\cdot)$ of (1) satisfies $\lim _{t \rightarrow+\infty} \mathbf{E}\left[x(t)^{\prime} x(t)\right]=0$. System (1) is called (mean-square) stablizable if there exists a stabilizing feedback control $u(t)=K x(t)$ where $K$ is a constant matrix.

Remark 2.1. The mean-square stabilizability can be conveniently verified by the following linear matrix inequality (LMI) condition:

$$
\left[\begin{array}{cc}
A X+X A^{\prime}+B Y+Y^{\prime} B^{\prime} & C X+D Y \\
X C^{\prime}+Y^{\prime} D^{\prime} & -X
\end{array}\right]<0 .
$$

Precisely, the controlled system is mean-square stabilizable if and only if (2) is feasible (with respect to the variables $X>0$ and $Y$ ) and the feedback control law $u(t)=Y X^{-1} x(t)$ is one stabilizing control; see [1, Theorem 2.1].

Definition 2.1. For a given $x_{0} \in \mathbf{R}^{n}$, the set of admissible controls is defined as follows:

$$
\mathbf{U}_{a d}\left(x_{0}\right) \triangleq\left\{u(\cdot) \in L_{2}^{\text {loc }}\left(\mathbf{R}^{n_{u}}\right) \mid u(\cdot) \text { is mean-square stabilizing w.r.t. } x_{0}\right\} \text {. }
$$

Throughout this paper we assume that system (1) is stabilizable. Hence $\mathbf{U}_{a d}\left(x_{0}\right)$ is nonempty for any $x_{0}$. It is easily seen that $\mathbf{U}_{a d}\left(x_{0}\right)$ is a convex subset of $L_{2}^{\text {loc }}\left(\mathbf{R}^{n_{u}}\right)$.

For each $\left(x_{0}, u(\cdot)\right) \in \mathbf{R}^{n} \times \mathbf{U}_{a d}\left(x_{0}\right)$ the associated cost function is defined as

$$
J\left(x_{0}, u(\cdot)\right)=\mathbf{E} \int_{0}^{+\infty}\left[x(t)^{\prime} Q x(t)+2 x(t)^{\prime} L u(t)+u(t)^{\prime} R u(t)\right] \mathrm{d} t,
$$

where $L \in \mathbf{R}^{n \times n_{u}}, Q \in \mathscr{S}^{n}, R \in \mathscr{S}^{n_{u}}$ are given matrices. The optimal control problem is to minimize the cost functional $J\left(x_{0}, u(\cdot)\right)$, for every $x_{0} \in \mathbf{R}^{n}$, over $u(\cdot) \in \mathbf{U}_{a d}\left(x_{0}\right)$. The value function $V$ is defined as

$$
V\left(x_{0}\right)=\inf _{u(\cdot) \in \mathbf{U}_{a d}\left(x_{0}\right)} J\left(x_{0}, u(\cdot)\right) .
$$

The LQ problem is called well-posed if

$$
V\left(x_{0}\right)>-\infty, \quad \forall x_{0} \in \mathbf{R}^{n} .
$$

Any control $u^{*}(\cdot)$ that achieves the infimum in (5) is called optimal (w.r.t. $x_{0}$ ). In this case, the LQ problem is called attainable (w.r.t. $x_{0}$ ).

The above problem is referred to as a (stochastic) linear-quadratic (LQ) problem. Since the symmetric matrix

$$
\left[\begin{array}{ll}
Q & L \\
L^{\prime} & R
\end{array}\right]
$$

is allowed to be indefinite, it is also called an indefinite LQ problem. Notice that $J\left(x_{0}, u(\cdot)\right)$ is not necessarily convex in $u(\cdot)$ due to the indefiniteness of the matrix (7).

\subsection{Generalized algebraic Riccati equation}

First, we recall some properties of the pseudo-inverse [13]. For any matrix $M$ there exists a unique matrix $M^{\dagger}$ satisfying

$$
M M^{\dagger} M=M, \quad M^{\dagger} M M^{\dagger}=M^{\dagger}, \quad\left(M M^{\dagger}\right)^{\prime}=M M^{\dagger}, \quad\left(M^{\dagger} M\right)^{\prime}=M^{\dagger} M .
$$


It can be easily seen (by singular value decomposition) that for any symmetric matrix $M$ the following hold:

$$
M^{\dagger}=M^{\dagger^{\prime}} ; \quad M M^{\dagger}=M^{\dagger} M ; \quad M \geqslant 0 \text { iff } M^{\dagger} \geqslant 0 ; \quad \text { and } \quad M^{1 / 2}\left(M^{1 / 2}\right)^{\dagger}=M M^{\dagger} \text { for } M \geqslant 0 .
$$

Definition 2.2. Let $(A, B, C, D, L) \in \mathbf{R}^{n \times n} \times \mathbf{R}^{n \times n_{u}} \times \mathbf{R}^{n \times n} \times \mathbf{R}^{n \times n_{u}} \times \mathbf{R}^{n \times n_{u}}$ and $(Q, R) \in \mathscr{S}^{n} \times \mathscr{S}^{n_{u}}$ be given constant matrices. The following constrained nonlinear algebraic equation

$$
\begin{aligned}
& A^{\prime} P+P A+C^{\prime} P C+Q-\left(P B+C^{\prime} P D+L\right)\left(R+D^{\prime} P D\right)^{\dagger}\left(B^{\prime} P+D^{\prime} P C+L^{\prime}\right)=0, \\
& {\left[I-\left(R+D^{\prime} P D\right)\left(R+D^{\prime} P D\right)^{\dagger}\right]\left(B^{\prime} P+D^{\prime} P C+L^{\prime}\right)=0,} \\
& R+D^{\prime} P D \geqslant 0,
\end{aligned}
$$

with the unknown $P \in \mathscr{S}^{n}$, is called a generalized algebraic Riccati equation (GARE).

For notational convenience, we use the following throughout this paper:

$$
\mathscr{M}(P) \triangleq A^{\prime} P+P A+C^{\prime} P C+Q, \quad \mathscr{L}(P) \triangleq P B+C^{\prime} P D+L, \quad \mathscr{N}(P) \triangleq R+D^{\prime} P D .
$$

In particular, if $R+D^{\prime} P D$ is nonsingular, then the pseudo-inverse becomes the standard inverse and the second constraint in (10) is redundant. In this case, GARE (10) reduces to the one considered in [1]:

$$
\begin{aligned}
& \mathscr{M}(P)-\mathscr{L}(P) \mathscr{N}(P)^{-1} \mathscr{L}(P)^{\prime}=0, \\
& \mathscr{N}(P)>0 .
\end{aligned}
$$

Another interesting special case is $R+D^{\prime} P D=0$, where GARE (10) becomes a system of algebraic linear equations

$$
\mathscr{M}(P)=0, \quad \mathscr{L}(P)=0, \quad \mathscr{N}(P)=0 .
$$

Definition 2.3. A solution $P$ to GARE (10) is said to be a maximal solution if $P \geqslant \tilde{P}$ for any $\tilde{P}$ with

$$
\begin{aligned}
& \mathscr{M}(\tilde{P})-\mathscr{L}(\tilde{P}) \mathscr{N}(\tilde{P})^{\dagger} \mathscr{L}(\tilde{P})^{\prime} \geqslant 0, \\
& {\left[I-\mathscr{N}(\tilde{P})^{\dagger} \mathscr{N}(\tilde{P})\right] \mathscr{L}(\tilde{P})^{\prime}=0,} \\
& \mathscr{N}(\tilde{P}) \geqslant 0 .
\end{aligned}
$$

The following lemma will be frequently used in this paper.

Lemma 2.1. Let $S \in \mathscr{S}^{n}$ be given. Then for any admissible pair $(x(\cdot), u(\cdot))$ of system (1), we have

$$
\mathbf{E}\left[x\left(t_{2}\right)^{\prime} S x\left(t_{2}\right)-x\left(t_{1}\right)^{\prime} S x\left(t_{1}\right)\right]-\mathbf{E} \int_{t_{1}}^{t_{2}}\left[x^{\prime}(\mathscr{M}(S)-Q) x+2 x^{\prime}(\mathscr{L}(S)-L) u+u^{\prime} D^{\prime} S D u\right](t) \mathrm{d} t=0 .
$$

Proof. This is immediate by applying Itô's formula to $x(t)^{\prime} S x(t)$.

The following is the main result of this section.

Theorem 2.1. Assume that GARE (10) has a solution $P$ and there exist $Y(\cdot) \in L_{2}^{\text {loc }}\left(\mathbf{R}^{n_{u} \times n}\right)$ and $z(\cdot) \in$ $L_{2}^{\text {loc }}\left(\mathbf{R}^{n_{u}}\right)$ such that the following control:

$$
u_{Y, z}(t)=-\left[\mathscr{N}(P)^{\dagger} \mathscr{L}(P)^{\prime}+\left(I-\mathscr{N}(P)^{\dagger} \mathscr{N}(P)\right) Y(t)\right] x(t)-\left[I-\mathscr{N}(P)^{\dagger} \mathscr{N}(P)\right] z(t)
$$

is admissible with respect to any initial $x_{0}$. Then the stochastic LQ problem (1)-(5) is well-posed and $u_{Y, z}(\cdot)$ is indeed an optimal control. Moreover, the value function is $V\left(x_{0}\right)=x_{0}^{\prime} P x_{0}$.

Proof. First of all, applying Lemma 2.1, we can express the cost function as follows:

$$
J\left(x_{0} ; u(\cdot)\right)=x_{0}^{\prime} P x_{0}+\mathbf{E} \int_{0}^{+\infty}\left[x^{\prime} \mathscr{M}(P) x+2 x^{\prime} \mathscr{L}(P) u+u^{\prime} \mathscr{N}(P) u\right](t) \mathrm{d} t, \quad \forall u(\cdot) \in \mathbf{U}_{a d}\left(x_{0}\right) .
$$


Define

$$
L_{1}(t)=Y(t)-\mathscr{N}(P)^{\dagger} \mathscr{N}(P) Y(t), \quad L_{2}(t)=z(t)-\mathscr{N}(P)^{\dagger} \mathscr{N}(P) z(t) .
$$

Using the properties (8) and (9) of the pseudo-inverse we conclude that $L_{1}$ and $L_{2}$ satisfy

$$
\mathscr{N}(P) L_{i}(t)=\mathscr{N}(P)^{\dagger} L_{i}(t)=0, \quad \mathscr{L}(P) L_{i}(t)=0, \quad i=1,2 .
$$

Then equality (17) is equivalent to

$$
\begin{aligned}
J\left(x_{0} ; u(\cdot)\right)= & x_{0}^{\prime} P x_{0}+\mathbf{E} \int_{0}^{+\infty}\left\{x^{\prime}\left[\mathscr{M}(P)-\mathscr{L}(P) \mathscr{N}(P)^{\dagger} \mathscr{L}(P)^{\prime}\right] x\right. \\
& \left.+\left[u+\left(\mathscr{N}(P)^{\dagger} \mathscr{L}(P)^{\prime}+L_{1}\right) x+L_{2}\right]^{\prime} \mathscr{N}(P)\left[u+\left(\mathscr{N}(P)^{\dagger} \mathscr{L}(P)^{\prime}+L_{1}\right) x+L_{2}\right]\right\}(t) \mathrm{d} t \\
= & x_{0}^{\prime} P x_{0}+\mathbf{E} \int_{0}^{+\infty}\left\{\left[u+\left(\mathscr{N}(P)^{\dagger} \mathscr{L}(P)^{\prime}+L_{1}\right) x+L_{2}\right]^{\prime}\right. \\
& \left.\mathscr{N}(P)\left[u+\left(\mathscr{N}(P)^{\dagger} \mathscr{L}(P)^{\prime}+L_{1}\right) x+L_{2}\right]\right\}(t) \mathrm{d} t .
\end{aligned}
$$

As a consequence, $J\left(x_{0} ; u(\cdot)\right)$ is minimized by the control $u_{Y, z}(\cdot)$ given by (16) with the optimal value being $x_{0}^{\prime} P x_{0}$.

The above theorem suggests the following definition.

Definition 2.4. A solution $P$ to GARE (10) is called stabilizing if there exists an admissible control of the form (16). Moreover, $P$ is called static stabilizing if there exists a constant matrix $Y$ such that the feedback control law $u(t)=\mathscr{K}(P, Y) x(t)$ with the following feedback gain:

$$
\mathscr{K}(P, Y) \triangleq-\mathscr{N}(P)^{\dagger} \mathscr{L}(P)^{\prime}-\left[I-\mathscr{N}(P)^{\dagger} \mathscr{N}(P)\right] Y
$$

is admissible with respect to any $x_{0}$.

We have an equivalent LMI condition for the existence of static stabilizing solutions to GARE (10).

Theorem 2.2. A solution $P$ of GARE (10) is static stabilizing if and only if there exist a matrix $Z$ and a symmetric matrix $X$ such that

$$
\left[\begin{array}{c|c}
A_{P} X+X A_{P}^{\prime}+B\left[I-\mathscr{N}(P)^{\dagger} \mathscr{N}(P)\right] Z & C_{P} X+D\left[I-\mathscr{N}(P)^{\dagger} \mathscr{N}(P)\right] Z \\
\hline+Z\left[I-\mathscr{N}(P)^{\dagger} \mathscr{N}(P)\right] B & -X
\end{array}\right]<0
$$

where

$$
\begin{aligned}
& A_{P}=A-B \mathscr{N}(P)^{\dagger} \mathscr{L}(P)^{\prime}, \\
& C_{P}=C-D \mathscr{N}(P)^{\dagger} \mathscr{L}(P)^{\prime} .
\end{aligned}
$$

Proof. It follows immediately from (2) in Remark 2.1.

The following result concerns the uniqueness of a stabilizing solution to GARE (10).

Theorem 2.3. There is at most one stabilizing solution to GARE (10). Moreover, a stabilizing solution to (10) must be its maximal solution.

Proof. Let $P_{1}$ and $P_{2}$ be two stabilizing solutions to (10). Then Theorem 2.1 implies that $x_{0}^{\prime} P_{1} x_{0}=x_{0}^{\prime} P_{2} x_{0}$ for any $x_{0} \in \mathbf{R}^{n}$, leading to $P_{1}=P_{2}$. 
Next, let $P$ be the stabilizing solution to the GARE and $\tilde{P}$ be any symmetric matrix satisfying (14). Putting $\tilde{P}$ in (19) we have

$$
J\left(x_{0}, u(\cdot)\right) \geqslant x_{0}^{\prime} \tilde{P} x_{0} \quad \forall u(\cdot) \in \mathbf{U}_{a d}\left(x_{0}\right) .
$$

Taking infimum over $u(\cdot) \in \mathbf{U}_{a d}\left(x_{0}\right)$ and applying Theorem 2.1, we have $x_{0}^{\prime} P x_{0}=V\left(x_{0}\right) \geqslant x_{0}^{\prime} \tilde{P} x_{0}$. This completes the proof.

Remark 2.2. Although there may be infinitely many optimal controls (which by definition are all mean-square stabilizing), there is at most one stabilizing solution of GARE (10), as in the standard (definite) LQ case.

Corollary 2.1. Let $P$ be a stabilizing solution to GARE (10). Then optimal controls of LQ problem (1)-(5) are obtained in the following special cases:

(i) If $R+D^{\prime} P D=0$, then any admissible control is optimal.

(ii) If $R+D^{\prime} P D>0$, then there is a unique optimal control that is given by $u(t)=-\mathscr{N}(P)^{-1} \mathscr{L}(P)^{\prime} x(t)$.

Proof. To prove (i), let $P$ be the stabilizing solution to (10) such that $R+D^{\prime} P D=0$. Using Lemma 2.1 we have

$$
J\left(x_{0} ; u(\cdot)\right)=x_{0}^{\prime} P x_{0}+\mathbf{E} \int_{0}^{+\infty}\left[x^{\prime} \mathscr{M}(P) x+2 u^{\prime} \mathscr{L}(P) x+u^{\prime} \mathscr{N}(P) u\right](t) \mathrm{d} t=x_{0}^{\prime} P x_{0}, \quad \forall u(\cdot) \in \mathbf{U}_{a d}\left(x_{0}\right) .
$$

Hence $J\left(x_{0} ; u(\cdot)\right)=V\left(x_{0}\right), \forall u(\cdot) \in \mathbf{U}_{a d}\left(x_{0}\right)$. This proves the desired result. On the other hand, (ii) is immediate by Theorem 2.1 .

\section{Well-posedness of LQ problem}

The aim of this section is to investigate the well-posedness of the LQ problem via an LMI condition. First, the following technical result can be shown by a simple adaptation of the well-known result in the deterministic case (see, e.g., [3]).

Lemma 3.1. LQ problem (5) is well-posed if and only if there exists a unique constant symmetric matrix $P$ such that

$$
V\left(x_{0}\right)=x_{0}^{\prime} P x_{0} \quad \forall x_{0} \in \mathbf{R}^{n} .
$$

Now, consider the following convex set of constant symmetric matrices

$$
\mathscr{P} \triangleq\left\{P \in \mathscr{S}^{n} \mid\left[\begin{array}{c|c}
\mathscr{M}(P) & \mathscr{L}(P) \\
\hline \mathscr{L}(P)^{\prime} & \mathscr{N}(P)
\end{array}\right] \geqslant 0\right\} .
$$

Theorem 3.1. $L Q$ problem (1)-(5) is well-posed if and only if $\mathscr{P} \neq \varnothing$. In this case, $\mathscr{P}$ has a maximal element $P \in \mathscr{P}$ (i.e., $P \geqslant \tilde{P} \forall \tilde{P} \in \mathscr{P})$. Moreover, we have

$$
V\left(x_{0}\right)=x_{0}^{\prime} P x_{0} \quad \forall x_{0} \in \mathbf{R}^{n} .
$$

Proof. Suppose $\mathscr{P} \neq \varnothing$. Let $\tilde{P}$ be any element of $\mathscr{P}$. Then (17) yields

$$
\begin{aligned}
J\left(x_{0}, u(\cdot)\right) & =x_{0}^{\prime} \tilde{P} x_{0}+\mathbf{E} \int_{0}^{+\infty}\left(\begin{array}{l}
x \\
u
\end{array}\right)^{\prime}\left[\begin{array}{l|l}
\mathscr{M}(\tilde{P}) & \mathscr{L}(\tilde{P}) \\
\hline \mathscr{L}(\tilde{P})^{\prime} & \mathscr{N}(\tilde{P})
\end{array}\right]\left(\begin{array}{l}
x \\
u
\end{array}\right)(t) \mathrm{d} t \\
& \geqslant x_{0}^{\prime} \tilde{P} x_{0} \quad \forall\left(x_{0}, u(\cdot)\right) \in \mathbf{R}^{n} \times \mathbf{U}_{a d}\left(x_{0}\right) .
\end{aligned}
$$

This implies that the original LQ problem is well-posed and

$$
V\left(x_{0}\right) \geqslant x_{0} \tilde{P} x_{0} .
$$


Conversely, assume that the LQ problem is well-posed. Then Lemma 3.1 implies the existence of a symmetric matrix $P$ such that $V\left(x_{0}\right)=x_{0}^{\prime} P x_{0}, \forall x_{0} \in \mathbf{R}^{n}$. Observe that if $\mathscr{P} \neq \varnothing$ then it follows from (25) that $P \geqslant \tilde{P}$, $\forall \tilde{P} \in \mathscr{P}$. Hence, the proof will be complete if we can show that $P \in \mathscr{P}$. To this end, we apply the dynamic programming principle to get

$$
x_{0}^{\prime} P x_{0} \leqslant \mathbf{E}\left\{\int_{0}^{h}\left[x(t)^{\prime} Q x(t)+2 x(t)^{\prime} L u(t)+u(t)^{\prime} R u(t)\right] \mathrm{d} t+x(h)^{\prime} P x(h)\right\}, \quad \forall h \geqslant 0, \quad \forall u(\cdot) \in \mathbf{U}_{a d}\left(x_{0}\right) .
$$

Applying Ito's formula to $x(t)^{\prime} P x(t)$, using the above inequality, and employing Lemma 2.1, we obtain

$$
\mathbf{E} \int_{0}^{h}\left(\begin{array}{l}
x \\
u
\end{array}\right)^{\prime}\left[\begin{array}{c|c}
\mathscr{M}(P) & \mathscr{L}(P) \\
\hline \mathscr{L}(P)^{\prime} & \mathscr{N}(P)
\end{array}\right]\left(\begin{array}{l}
x \\
u
\end{array}\right)(t) \mathrm{d} t \geqslant 0, \quad \forall u(\cdot) \in \mathbf{U}_{a d}\left(x_{0}\right) .
$$

Dividing both sides by $h$ and letting $h \rightarrow 0$, we obtain

$$
\left(\begin{array}{l}
x(0) \\
u(0)
\end{array}\right)^{\prime}\left[\begin{array}{c|c}
\mathscr{M}(P) & \mathscr{L}(P) \\
\hline \mathscr{L}(P)^{\prime} & \mathscr{N}(P)
\end{array}\right]\left(\begin{array}{c}
x(0) \\
u(0)
\end{array}\right) \geqslant 0 .
$$

Since $x(0) \in \mathbf{R}^{n}$ and $u(0) \in \mathbf{R}^{n_{u}}$ can be chosen arbitrarily, we conclude

$$
\left[\begin{array}{l|l}
\mathscr{M}(P) & \mathscr{L}(P) \\
\hline \mathscr{L}(P)^{\prime} & \mathscr{N}(P)
\end{array}\right] \geqslant 0
$$

namely, $P \in \mathscr{P}$.

Remark 3.1. The above result gives a very convenient way, in particular computationally, of verifying the well-posedness of an indefinite LQ problem. Specifically, one only need to solve the LMI condition in (23), for which many efficient solvers exist; see $[5,8,15]$.

\section{Attainability of LQ problem}

This section further characterizes the attainability of the LQ problem in terms of GARE (10). First, we present some lemmas required in the proof of the main result.

Lemma 4.1 (Extended Schur's Lemma [2]). Let matrices $S=S^{\prime}, T=T^{\prime}$ and $U$ be given with appropriate sizes. Then the following conditions are equivalent:

(i) $S-U T^{\dagger} U^{\prime} \geqslant 0, T \geqslant 0$, and $\left(I-T T^{\dagger}\right) U^{\prime}=0$.

(ii) $\left[\begin{array}{cc}S & U \\ U^{\prime} & T\end{array}\right] \geqslant 0$.

Lemma 4.2. Let matrices $L, M$ and $N$ be given with appropriate sizes. Then the following matrix equation

$$
L X M=N
$$

has a solution $X$ if and only if

$$
L L^{\dagger} N M^{\dagger} M=N \text {. }
$$

Moreover, any solution to (27) is represented by

$$
X=L^{\dagger} N M^{\dagger}+S-L^{\dagger} L S M M^{\dagger},
$$

where $S$ is a matrix with an appropriate size.

Proof. The basic idea of the following proof is due to [2, Theorem 1(i)]; we reproduce the proof here for reader's convenience. 
If $X$ satisfies the equation $L X M=N$, then we have

$$
N=L X M=L L^{\dagger} L X M M^{\dagger} M=L L^{\dagger} N M^{\dagger} M \text {. }
$$

Conversely, if (28) is satisfied, then $L^{\dagger} N M^{\dagger}$ is a solution of $L X M=N$. This proves the first part of the lemma. Now, let $Y$ be any matrix with appropriate size and define $\tilde{X}=Y-L^{\dagger} L Y M M^{\dagger}$. Then $\tilde{X}$ satisfies the homogeneous equation $L \tilde{X} M=0$. Hence $L^{\dagger} N M^{\dagger}+\tilde{X}$ must satisfy (27). On the other hand, let $X$ be a solution to (27). Then by (28), one has $L S M=0$ where $S=X-L L^{\dagger} N M^{\dagger} M$. Hence

$$
X=L L^{\dagger} N M^{\dagger} M+S \equiv L L^{\dagger} N M^{\dagger} M+S-L^{\dagger} L S M M^{\dagger} \text {. }
$$

This completes the proof.

The following theorem can be regarded as the converse of Theorem 2.1.

Theorem 4.1. If LQ problem (1)-(5) is attainable w.r.t. any initial $x_{0}$, then GARE (10) has a stabilizing solution. Moreover, any optimal control law is of the form (16) for some $Y(\cdot) \in L_{2}^{\text {loc }}\left(\mathbf{R}^{n_{u} \times n}\right)$ and $z(\cdot) \in$ $L_{2}^{\text {loc }}\left(\mathbf{R}^{n_{u}}\right)$.

Proof. First, we prove that (10) has a solution. Since the LQ problem is attainable, and hence well-posed, Theorem 3.1 yields that the set $\mathscr{P}$ has a maximal element $P$ such that $V\left(x_{0}\right)=x_{0}^{\prime} P x_{0}$, and

$$
\left[\begin{array}{c|c}
\mathscr{M}(P) & \mathscr{L}(P) \\
\hline \mathscr{L}(P)^{\prime} & \mathscr{N}(P)
\end{array}\right] \geqslant 0 .
$$

Applying Lemma 4.1 to (30), we have

$$
\begin{aligned}
& \mathscr{M}(P)-\mathscr{L}(P) \mathscr{N}(P)^{\dagger} \mathscr{L}(P)^{\prime} \geqslant 0, \\
& {\left[I-\mathscr{N}(P) \mathscr{N}(P)^{\dagger}\right] \mathscr{L}(P)^{\prime}=0,} \\
& \mathscr{N}(P) \geqslant 0 .
\end{aligned}
$$

Now, let $\left(x_{*}(\cdot), u_{*}(\cdot)\right)$ be an optimal state-control pair with respect to the initial condition $x(0)=x_{0}$. Applying Lemma 2.1 to $P$ and adding this to $J\left(x_{0}, u_{*}(\cdot)\right)$, we have

$$
\begin{aligned}
V\left(x_{0}\right) \equiv J\left(x_{0}, u_{*}(\cdot)\right)= & x_{0}^{\prime} P x_{0}+\mathbf{E} \int_{0}^{+\infty}\left[x_{*}^{\prime}\left(\mathscr{M}(P)-\mathscr{L}(P) \mathscr{N}(P)^{\dagger} \mathscr{L}(P)^{\prime}\right) x_{*}\right](t) \mathrm{d} t \\
& +\mathbf{E} \int_{0}^{+\infty}\left[u_{*}+\mathscr{N}(P)^{\dagger} \mathscr{L}(P)^{\prime} x_{*}\right]^{\prime} \mathscr{N}(P)\left[u_{*}+\mathscr{N}(P)^{\dagger} \mathscr{L}(P)^{\prime} x_{*}\right](t) \mathrm{d} t .
\end{aligned}
$$

Due to the equality $V\left(x_{0}\right)=x_{0}^{\prime} P x_{0}$ and (31)-(32), each of the two integrands on the right-hand side must be zero almost everywhere. Hence, we obtain

$$
\mathscr{M}(P)-\mathscr{L}(P) \mathscr{N}(P)^{\dagger} \mathscr{L}(P)^{\prime}=0,
$$

which, along with (31), implies that $P$ is a solution to GARE (10).

It remains to show that any optimal control $u^{*}(\cdot)$ can be represented by (16) for some $Y(\cdot)$ and $z(\cdot)$. From (32) it follows that

$$
\mathscr{N}(P)^{1 / 2}\left[u_{*}(t)+\mathscr{N}(P)^{\dagger} \mathscr{L}(P)^{\prime} x_{*}(t)\right]=0,
$$

which gives

$$
\mathscr{N}(P) u_{*}(t)+\mathscr{L}(P)^{\prime} x_{*}(t)=0, \quad \text { a.e. } \quad t \in[0,+\infty) .
$$

To solve the above equation with the unknown $u_{*}(t)$, we apply Lemma 4.2 with

$$
L=\mathscr{N}(P), \quad M=I, \quad N=-\mathscr{L}(P)^{\prime} x_{*}(t) .
$$

Notice that condition (28) in the present case is implied by the second constraint in GARE (10), hence general solution (29) with $z(t)=S$ and $Y(t)=0$ yields that $u(t)$ can be represented by (16). This also shows that $P$ is a stabilizing solution of (10). The proof is complete. 


\section{Relation to semidefinite programming}

In this section we show that any indefinite LQ problem can be turned into a convex optimization problem, called a semidefinite programming (SDP). This enables us to use existing SDP algorithms [5,15] to computationally obtain the optimal controls and value function of the LQ problem as well as the maximal solution to the GARE if such a solution exists.

To proceed, define the following SDP:

$$
\begin{aligned}
& \operatorname{minimize}-\mathbf{T r}(P), \\
& \text { subject to }\left[\begin{array}{l|l}
\mathscr{M}(P) & \mathscr{L}(P) \\
\hline \mathscr{L}(P)^{\prime} & \mathscr{N}(P)
\end{array}\right] \geqslant 0 .
\end{aligned}
$$

\section{Theorem 5.1. The following hold:}

(i) SDP (34) is feasible if and only if LQ problem (1)-(5) is well-posed.

(ii) If (34) is feasible then it has a unique optimal solution $P^{*}$ with $V\left(x_{0}\right)=x_{0}^{\prime} P^{*} x_{0} \forall x_{0} \in \mathbf{R}^{n}$.

(iii) If LQ problem (1)-(5) is attainable then the unique optimal solution of (34) is the stabilizing solution to GARE (10).

Proof. (i) It follows immediately from Theorem 3.1.

(ii) By Theorem 3.1 the feasible set of (34) has a maximal element $P$, which is obviously an optimal solution to (34). To show the uniqueness, let $P^{*}$ be any optimal solution to (34). Then by optimality we have $\operatorname{Tr}\left(P-P^{*}\right)=0$. On the other hand, the maximality of $P$ implies $P-P^{*} \geqslant 0$. Now, $P-P^{*}$ is a positive matrix with a null trace, so it has to be identically zero. Finally, by Theorem 3.1 we have $V\left(x_{0}\right)=x_{0}^{\prime} P x_{0}$.

(iii) This is an immediate consequence of Theorem 2.3 and Theorem 4.1.

We have noticed that for a stochastic LQ problem, $R$ may be indefinite (negative definite, in particular) while the LQ problem is still well-posed. A natural but interesting question is: How negative $R$ can be before the LQ problem becomes ill-posed? The following presents a numerical way of determining the "permissible negative range" of $R$.

Definition 5.1. Let $Q=Q^{\prime}$ and $L$ be given. The wellposedness margin $r^{*}$ is defined as the smallest scalar $r \in \mathbf{R}$ such that the LQ problem is well-posed for any symmetric matrix $R \geqslant r^{*} I$.

The wellposedness margin $r^{*}$ has the following interpretation:

- If the smallest eigenvalue of $R, \lambda_{\min }(R)$, is such that $\lambda_{\min }(R) \geqslant r^{*}$, then the LQ problem is well-posed.

- If the largest eigenvalue of $R, \lambda_{\max }(R)$, is such that $\lambda_{\max }(R)<r^{*}$, then the LQ problem is ill-posed.

- If $r^{*}=0$, the LQ problem is ill-posed with any $R$ having at least one negative eigenvalue.

Theorem 5.2. The well-posedness margin $r^{*}$ is the optimal solution to the following SDP:

$$
\begin{array}{ll|c}
\text { minimize } & r \\
\text { subject to } & \left\{\left[\begin{array}{c|c}
A^{\prime} P+P A+C^{\prime} P C+Q & P B+C^{\prime} P D+L \\
\hline B^{\prime} P+D^{\prime} P C+L & D^{\prime} P D+r I
\end{array}\right] \geqslant 0 .\right.
\end{array}
$$

Moreover, the optimum is always achievable by $r^{*}>-\infty$.

Proof. The first part of the result is evident from Theorem 5.1. To prove the second one, first observe that the value function as a function of $R$,

$$
V\left(x_{0}, R\right)=\inf _{u(\cdot) \in \mathbf{U}_{a d}\left(x_{0}\right)} \mathbf{E} \int_{0}^{+\infty}\left[x(t)^{\prime} Q x(t)+2 x(t)^{\prime} L u(t)+u(t)^{\prime} R u(t)\right] \mathrm{d} t
$$


is monotone, namely,

$$
V\left(x_{0}, R_{1}\right) \geqslant V\left(x_{0}, R_{2}\right) \quad \forall x_{0} \in \mathbf{R}^{n}, \quad \text { if } R_{1} \geqslant R_{2} .
$$

Now, assume that the optimal solution of (35) $r^{*}=-\infty$. Then by definition the LQ problem is well-posed for any $R=r I, r \in(-\infty, 0]$. By Theorem 3.1, there exists a sequence of a symmetric matrices $P_{r}$, decreasing as $r$ decreases to $-\infty$, such that

$$
V\left(x_{0}, r I\right)=x_{0} P_{r} x_{0}, \quad D^{\prime} P_{r} D+r I \geqslant 0 .
$$

Set $\alpha_{r}=\operatorname{Tr}\left(D^{\prime} P_{r} D\right) \geqslant-\operatorname{Tr}(r I) \geqslant 0$. Then $\alpha_{r}$ is a decreasing positive sequence, so $\lim _{r \rightarrow-\infty} \alpha_{r}$ exists and is finite. But this contradicts to the fact that $\alpha_{r} \geqslant-n r$.

\section{Examples}

In this section we present two examples illustrating the results obtained. In the first example, the LQ problem is well-posed but not attainable, while in the second example the problem is attainable.

Example 6.1. Consider a two-dimensional LQ problem with the following data in the system dynamics (1):

$$
A=\left[\begin{array}{rr}
-10 & 5 \\
0 & 3
\end{array}\right], \quad B=\left[\begin{array}{ll}
1 & 0 \\
0 & 1
\end{array}\right], \quad C=\left[\begin{array}{ll}
1 & 0 \\
0 & 0
\end{array}\right], \quad D=\left[\begin{array}{ll}
1 & 0 \\
0 & 0
\end{array}\right] .
$$

First, we show that the system is mean-square stabilizable. By Remark 2.1, we only need to check if the LMI condition (2) (with respect to the variables $X$ and $Y$ ) has a solution. It turns out that

$$
X=\left[\begin{array}{cc}
1185.4133 & 0 \\
0 & 2974.8354
\end{array}\right], \quad Y=\left[\begin{array}{cc}
501.79932 & 0 \\
-5927.0666 & -10411.924
\end{array}\right] .
$$

Next, we consider the LQ problem with the following cost weighting matrices:

$$
Q=\left[\begin{array}{rr}
54 & -10 \\
-10 & 0
\end{array}\right], \quad R=\left[\begin{array}{rr}
-1 & 0 \\
0 & 0
\end{array}\right]
$$

We can check that the symmetric matrix

$$
P=\left[\begin{array}{ll}
2 & 0 \\
0 & 0
\end{array}\right]
$$

satisfies GARE (10). Thus by Theorem 3.1 the LQ problem is well-posed. Moreover, $P$ is the maximal solution to (10), but not stabilizing (which is verified by the LMI condition in Theorem 2.2). It then follows from Theorems 4.1 and 2.3 that the LQ problem is not attainable.

Example 6.2. Take the same data as in Example 6.2 except now

$$
C=\left[\begin{array}{rr}
-1 & 0 \\
0 & 0
\end{array}\right]
$$

Then

$$
P=\left[\begin{array}{ll}
1 & 0 \\
0 & 0
\end{array}\right]
$$

is a static feedback stabilizing solution to GARE (10) by Theorem 2.2. Thus it follows from Theorems 2.1 and 4.1 that the LQ problem is attainable with all possible optimal control laws given by (16). 


\section{Conclusion}

This paper studied LQ models with multiplicative noises in signals and indefinite quadratic weights in costs. Such models, which are less restrictive than those in standard LQ theory, better approximate more general, nonlinear stochastic systems and arise naturally in areas of current interest such as in finance.

The well-posedness of an LQ problem has been characterized in terms of the existence of a solution to an LMI condition, and the existence of optimal controls has been understood in terms of the existence of a solution to a constrained Riccati equation involving a pseudo-inverse, which in turn is the vehicle for calculating all the optimal controls and the optimal value. These give rise to analytical as well as computational advantages to solving the LQ problem. It is also worth mentioning that even for the deterministic singular case, the results presented in this paper are stronger and more general than those in the literature. In particular, in the deterministic case, GARE (10) reduces to

$$
\begin{aligned}
& P A+A^{\prime} P-P B R^{\dagger} B^{\prime} P+Q=0, \\
& R R^{\dagger} B^{\prime} P-B^{\prime} P=0, \\
& R \geqslant 0,
\end{aligned}
$$

and optimal controls are given by

$$
u_{Y, z}(t)=\left[-R^{\dagger} B^{\prime} P+Y(t)-R^{\dagger} R Y(t)\right] x(t)+z(t)-R^{\dagger} R z(t)
$$

\section{Acknowledgements}

This research is supported in part by the RGC Earmarked Grants CUHK 4125/97E and CUHK 4054/98E.

\section{References}

[1] M. Ait Rami, X.Y. Zhou, Linear matrix inequalities, Riccati equations, and indefinite stochastic linear quadratic control, IEEE Trans. Automat. Control, to appear.

[2] A. Albert, Conditions for positive and nonnegative definiteness in terms of pseudo-inverses, SIAM J. Appl. Math. 17 (1969) $434-440$.

[3] B.D.O. Anderson, J.B. Moore, Optimal Control: Linear Quadratic Methods, Prentice-Hall, Englewood Cliffs, NJ, 1989.

[4] M. Athans, Special issues on linear-quadratic-Gaussian problem, IEEE Trans. Automat. Control AC-16 (1971) $527-869$.

[5] S. Boyd, L. El Ghaoui, E. Feron, V. Balakrishnan, Linear Matrix Inequality in Systems and Control Theory, SIAM, Philadelphia, PA, 1994.

[6] S. Chen, X. Li, X.Y. Zhou, Stochastic linear quadratic regulators with indefinite control weight costs, SIAM J. Control Optim. 36 (1998) 1685-1702.

[7] M.H.A. Davis, Linear Estimation and Stochastic Control, Chapman \& Hall, London, 1977.

[8] L. El Ghaoui, R. Nikoukhah, F. Delebecque, LMITOOL: a front-end for LMI optimization in Matlab, ENSTA, Paris, 1995.

[9] R.E. Kalman, Contribution to the theory of optimal control, Bol. Soc. Mat. Mex. 5 (1960) 102-119.

[10] M. Kohlmann, X.Y. Zhou, Relationship between backward stochastic differential equations and stochastic controls: a linear-quadratic approach, SIAM J. Control Optim. 38 (2000) 1392-1407.

[11] A.E.B. Lim, X.Y. Zhou, Optimal stochastic LQR control with integral quadratic constraints and indefinite control weights, IEEE Trans. Automat. Control 44 (1999) 359-369.

[12] J.B. Moore, X.Y. Zhou, A.E.B. Lim, LQG control of linear stochastic systems with control dependent noise, Syst. Control Lett. 36 (1999) 199-206.

[13] R. Penrose, A generalized inverse of matrices, Proc. Cambridge Philos. Soc. 52 (1955) 17-19.

[14] V.M. Popov, Hyperstability and optimality of automatic systems with several control functions, Rev. Roum. Sci. Tech., Ser. Electrotech. Energ. 9 (1964) 629-690.

[15] L. Vandenerghe, S. Boyd, Semidefinite programming, SIAM Rev. 38 (1996) 49-95.

[16] J.C. Willems, Least squares stationary control and the algebraic Riccati equation, IEEE Trans. Automat. Control 16 (1971) 621-634.

[17] W.M. Wonham, On a matrix Riccati equation of stochastic control, SIAM J. Control 6 (1968) 312-326.

[18] X.Y. Zhou, D. Li, Explicit efficient frontier of a continuous-time mean-variance portfolio selection problem, in: Chen et al. (Eds.), Control of Distributed Parameter and Stochastic Systems, Kluwer Academic Publishers, Boston, 1999, pp. 323-330. 